\title{
Thermal decomposition of solid mixtures of 2-oxy-4, 6-dinitramine-s-triazine (DNAM) and phase stabilized ammonium nitrate (PSAN)
}

\author{
P.N. Simões ${ }^{\mathrm{a}, *}$, L.M. Pedroso ${ }^{\mathrm{a}}$, A.A. Portugal ${ }^{\mathrm{a}}$, J.L. Campos ${ }^{\mathrm{b}}$ \\ ${ }^{a}$ Laboratório de Energética e Detónica, Departamento de Engenharia Química, Faculdade de \\ Ciências e Tecnologia Universidade de Coimbra, Coimbra, Portugal \\ ${ }^{\mathrm{b}}$ Laboratório de Energética e Detónica, Departamento de Engenharia Mecânica, Faculdade de \\ Ciências e Technologia, Universidade de Coimbra, Coimbra, Portugal
}

Received 7 January 1999; received in revised form 26 June 2000; accepted 2 July 2000

\begin{abstract}
The thermal decomposition of solid mixtures of 2-oxy-4,6-dinitramine-s-triazine (DNAM) and phase stabilized ammonium nitrate (PSAN) at different mass ratios has been studied. Simultaneous thermal analysis (DSC/TG) and thermomicroscopy have been used. It was found that PSAN promotes the lowering of the decomposition temperature of DNAM. The beginning of this process occurs when both components are in the solid state irrespective of the composition. However, the composition appears as the main factor determining the process progression once initiated. These observations are interpreted in the light of known properties of both DNAM and PSAN. Non-isothermal kinetic analysis, restricted to the early stage of the decomposition process, has been performed for the particular DNAM/PSAN ratios of 50/50 and 60/40. The complexity of the process is evidenced by the impossibility of being described by a kinetic model function other than an empirical one (SestakBerggren). Noticeable differences in the apparent Arrhenius parameters were found, indicating remarkable changes in the process over the composition range of the kinetic analysis. (C) 2000 Elsevier Science B.V. All rights reserved.
\end{abstract}

Keywords: Dinitroammeline (DNAM); Non-isothermal kinetic analysis; Phase stabilized ammonium nitrate (PSAN); Thermal decomposition; Thermomicroscopy

\section{Introduction}

Searching for new materials to be used in propellant formulations is a continuous challenge. Usually, high performances are constrained by safety considerations. Environmental restrictions also have to be taken into account, leading to the use of clean rocket propellants and naturally excluding halogenous com-

\footnotetext{
* Corresponding author. Tel.: +351-39-798732; fax: +351-39-798703.

E-mail address: pnsim@eq.uc.pt (P.N. Simões).
}

pounds. The compound 2-oxy-4,6-dinitramine-s-triazine (DNAM) has recently been proposed [1-3] as a new candidate for clean propellant formulations, namely in combination with phase stabilized ammonium nitrate (PSAN). Some interesting attributes exhibited by DNAM make it a worthwhile candidate for such a kind of compositions. It is a stable and safety chlorine-free material, with a fairly attractive density as well as with energetic potential. Among others well-known oxidizing components, ammonium nitrate (AN) or one of its phase stabilized forms (PSAN) have been widely used in virtue of its low 
cost, chemical stability, low sensitivity to friction and to shock, as well as its low polluting characteristics. Some efforts have successfully been made to reduce some of the drawbacks of AN, such as the high hygroscopicity, solid state phase transition characteristics, and particle shape. Nevertheless, to get around the intrinsic problem of the low energy and low reactivity of either AN or PSAN, other solutions are necessary, being the partial replacement by other energetic components a possibility. The thermal decomposition of AN has been widely studied [411], and some reported works on the particular cases of PSAN [12,13] and DNAM [1] are also available.

The present paper reports the study of the thermal decomposition under non-isothermal conditions of solid mixtures of DNAM and PSAN, for DNAM/ PSAN mass ratios ranging from $10 / 90$ to $90 / 10$. Thermomicroscopy has also been employed to elucidate some features of the process. The solid decomposition thermoanalytical data of the compositions with the DNAM/PSAN ratios of 50/50 and 60/40, that are close to the stoichiometrically balanced fuel-oxidizer mixture [14] of the two components, have been submitted to a kinetic analysis to get relevant information on the thermochemistry of the process.

\section{Experimental}

\subsection{Sample preparation}

DNAM was synthesized and purified according to a procedure described elsewhere [15]. The purity of the product was evaluated by elemental analysis (Fissons EA1180): Anal. Calc. for $\mathrm{C}_{3} \mathrm{H}_{3} \mathrm{~N}_{7} \mathrm{O}_{5}: \mathrm{C}, 16.58 ; \mathrm{H}$, 1.39; N, 45.16. Found: C, 16.72, 16.81; H, 1.45, 1.49; $\mathrm{N}, 45.06,44.82$. The particle size distribution was measured by a laser diffraction technique with PIDS (Coulter LS130) and a monomodal distribution was found with particle size mean diameter $\left(d_{50}\right)$ of $2.5 \mu \mathrm{m}$. Density was measured by gas (helium) picnometry (AccuPyc 1330) and a value of $1945 \mathrm{~kg} \mathrm{~m}^{-3}$ was obtained.

The PSAN used in this study was obtained from ICT, Germany. It contained $1.0 \% \mathrm{NiO}$, as phase stabilizing agent, and $0.5 \%$ Petro (sodiumlaurylsulfonate), as anti-caking agent. The measured value for density was $1716 \mathrm{~kg} \mathrm{~m}^{-3}$. The particle size distribution measurement was carried out in dry conditions (Malvern 2600C) and a particle median diameter of $169 \mu \mathrm{m}$ was obtained.

Pure AN and pure cyanuric acid (2,4,6-triol-s-triazine) supplied by Merck were also used (as received) in our study.

\subsection{Thermal analysis instruments, conditions and procedures}

Simultaneous thermal analysis (heat flux DSC and TGA) was employed to investigate the thermal decomposition of DNAM/PSAN mixtures in non-isothermal conditions. The measurements were carried out using a Rheometric Scientific STA 1500 equipment. Temperature calibration for both modules (DSC and TGA) were performed in the range $25-500^{\circ} \mathrm{C}$ by running melting standards such as indium, tin and lead. The same standards were used in order to obtain calorimetric results in the DSC module. The calibration were performed for each heating rate values used throughout the experimental work $(2.5,5,7.5$, 10 and $15^{\circ} \mathrm{C} \min ^{-1}$ ). The gain of the thermobalance was chosen to give an approximate resolution of $0.5 \mu \mathrm{g}$.

The influence of the composition on the thermal decomposition process was studied by running the DNAM/PSAN samples at different mass ratios: 10/90, 20/80, 30/70, 50/50, 60/40, 70/30, 80/20 and 90/10 at $10{ }^{\circ} \mathrm{Cmin}^{-1}$. At least two runs were performed for each composition. The kinetic study was restricted to compositions 50/50 and 60/40, for which three and two runs, respectively, were conducted at each heating rate (from 2.5 to $15^{\circ} \mathrm{C} \mathrm{min}^{-1}$ ). For all experiments, the sample mass was $(2.10 \pm 0.02 \mathrm{mg})$ (unless otherwise stated, all intervals are $95 \%$ confidence level). The samples, after being prepared in a dry atmosphere by mixing the components with the aid of a small spatula, were loaded into open alumina crucibles. A dry nitrogen purge flow of $50 \mathrm{ml} \mathrm{min}^{-1}$ at $0.1 \mathrm{MPa}$ absolute pressure was used in all measurements.

A control run was carried out in order to detect the possible influences of additives ( $\mathrm{NiO}$ and Petro) of PSAN on the decomposition process under study. This has been checked for a mixture of DNAM and dried

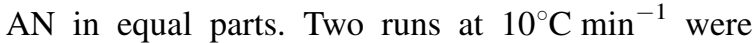
carried out. The thermal decomposition process did 
not exhibit any noticeable departure from the results obtained for PSAN runs.

Thermomicroscopy (Mettler Toledo FP9O Central Processor; Mettler Toledo FP84 HT Microscopy Cell; Leica DMRB microscope) was used for the 10/90, 30/ 70 and 50/50 mixtures to elucidate some features of the decomposition process at $10^{\circ} \mathrm{C} \mathrm{min}^{-1}$.

\section{Results and discussion}

\subsection{Thermal decomposition of DNAM/PSAN mixtures}

In Fig. 1 an overview of the thermal behavior of some DNAM/PSAN mixtures at $10^{\circ} \mathrm{C} \mathrm{min}^{-1}$ is presented. Fig. 2 gives more detailed heat flux DSC and TG curves in the restricted $100-300^{\circ} \mathrm{C}$ temperature range for all compositions tested. The thermoanalytical (TA) curves referring to each component alone are also presented for comparative purposes. Pure DNAM decomposes sharply above $220^{\circ} \mathrm{C}$ without any other thermal event prior to the decomposition. This pattern is in agreement with a recently reported study under comparable conditions on the thermal decomposition of DNAM [1]. A rather different thermal behavior is exhibited by PSAN, whose endothermic decomposition, that occurs in the approximate temperature range of $170-270^{\circ} \mathrm{C}$, is preceded by the phase transitions IV $\rightarrow$ II and II $\rightarrow$ I with peak temperatures at 60 and $125^{\circ} \mathrm{C}$, respectively, and melting starting approximately at $150^{\circ} \mathrm{C}$ [12].

DSC measurements (Figs. 1 and 2(a)) allowed us to conclude that the on-set and peak temperatures of the phase transitions IV $\rightarrow$ II and II $\rightarrow$ I of PSAN are not influenced by the presence of DNAM. Considering in detail the TA results in the temperature range from 100 to $300^{\circ} \mathrm{C}$ (Fig. 2), some effects are to be underlined. Considering the TG curves (Fig. 2(b)), no reactive interaction between the two components with gas evolution was detected before $125^{\circ} \mathrm{C}$. Nevertheless, a careful look into the TG curves permitted to conclude that the mass loss starts at temperatures close to the transition II $\rightarrow$ I. This means that the decomposition starts before the melting of PSAN, therefore when both components are still in the solid state. The proximity of the phase transition II $\rightarrow$ I event seems to be determinant for the beginning of the decomposition process in all mixtures considered, while the composition appears as the main factor determining the progress of the process once initiated. When the composition varies from $30 / 70$ to $50 / 50$, a remarkable change in the leading edge of the first exothermic peak is observed (Fig. 2(a)). This suggests that a turning point in the reactive process takes place in this composition range.

In order to clarify the effects underlying the TA curves, thermomicroscopy has been used to follow

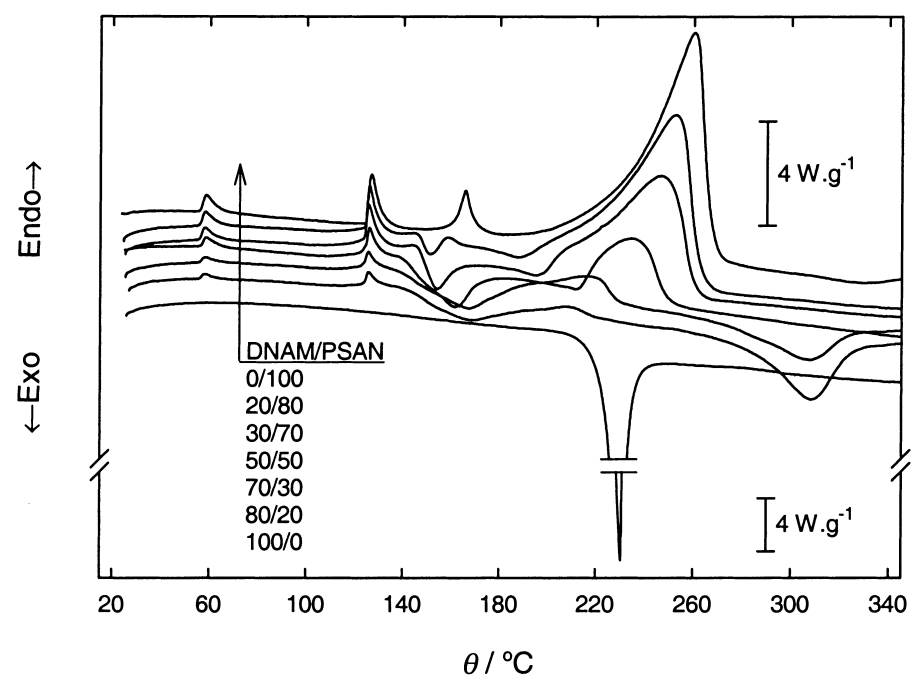

Fig. 1. DSC curves of DNAM, PSAN, and some DNAM/PSAN solid mixtures at $10^{\circ} \mathrm{C} \mathrm{min}^{-1}$. 

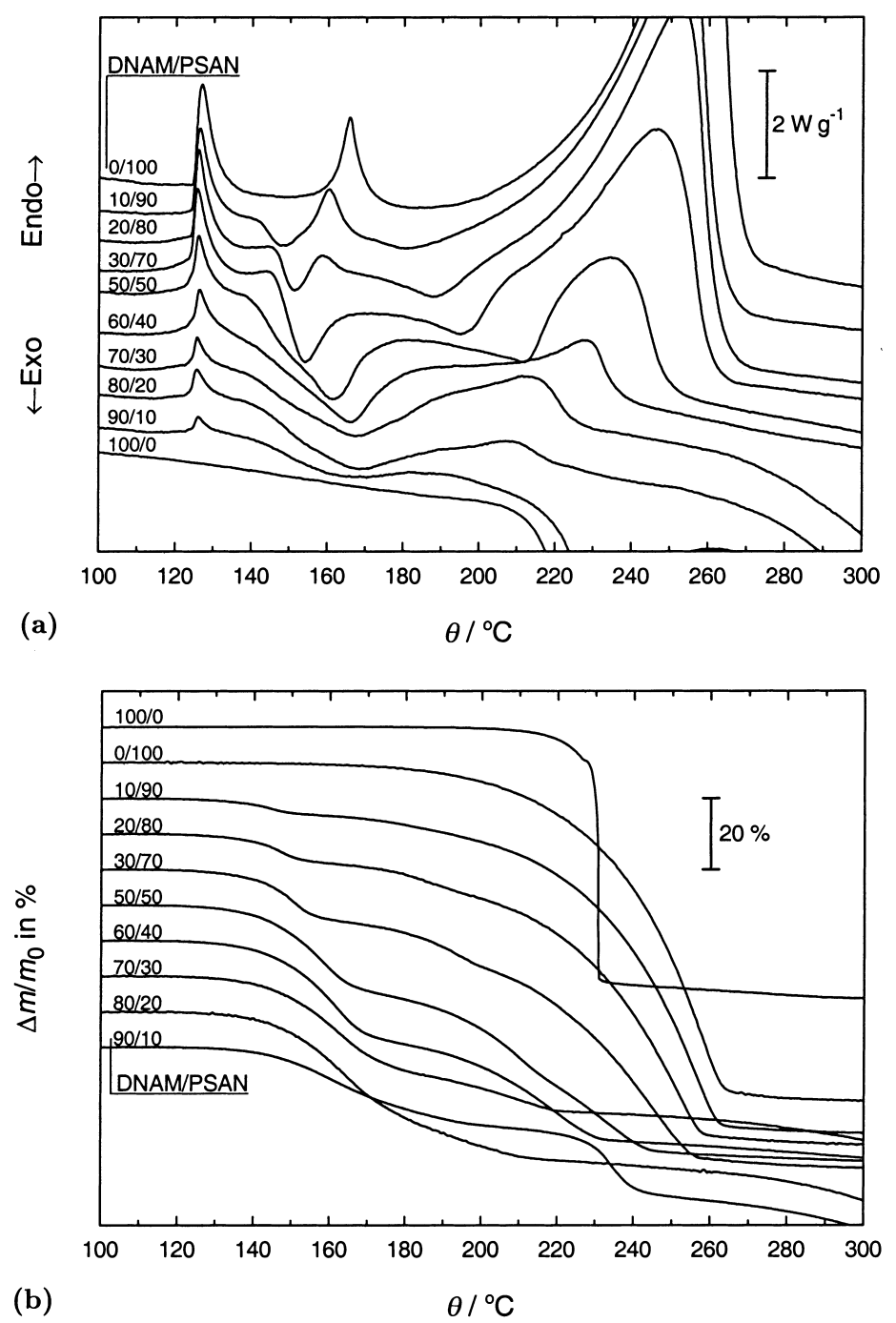

Fig. 2. Detailed view of (a) DSC and (b) TG curves of DNAM, PSAN, and all DNAM/PSAN solid mixtures studied at $10^{\circ} \mathrm{C} \mathrm{min}{ }^{-1}$.

the thermal behavior of the mixtures 10/90, 30/70 (see Fig. 3) and 50/50 under the heating rate of

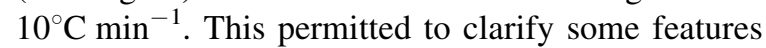
of the process as well as to open space for some inferences.

Thermomicroscopy allowed to confirm the start of decomposition before the phase transition II $\rightarrow$ I. Fig. 3 shows that some changes are detected in the mixture from $111^{\circ} \mathrm{C}$ to the onset $\left(124^{\circ} \mathrm{C}\right)$ of the transition II $\rightarrow$ I of PSAN (unlike the transition IV $\rightarrow$ II, a clear change of color of the PSAN particles helped to visually identify the last solid/solid phase transition of PSAN). The beginning of the reactive activity was evidenced not only by slight movements in some of the smallest particles, but also by noticeable changes in the shape of some of them. The differences in shape as well as in size of PSAN and DNAM particles helped to identify the later as the component that starts to decompose.

Pure DNAM decomposes without melting above $220^{\circ} \mathrm{C}$. Therefore, solid PSAN provides an environmental susceptible of lowering the onset temperature of the decomposition of DNAM, whose interpretation implies to consider how the components may physi- 


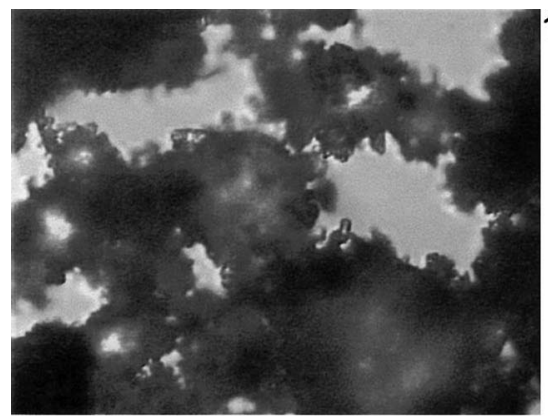

$111^{\circ} \mathrm{C}$
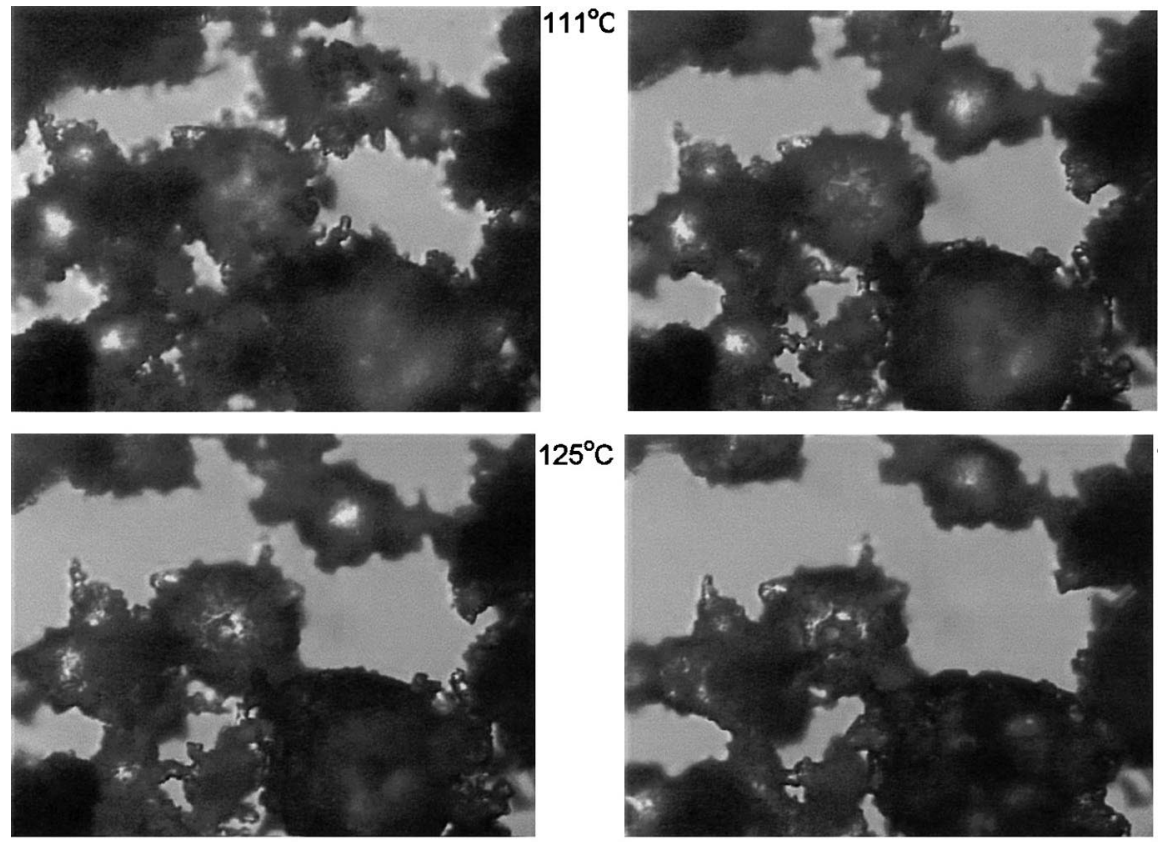

$125^{\circ} \mathrm{C}$
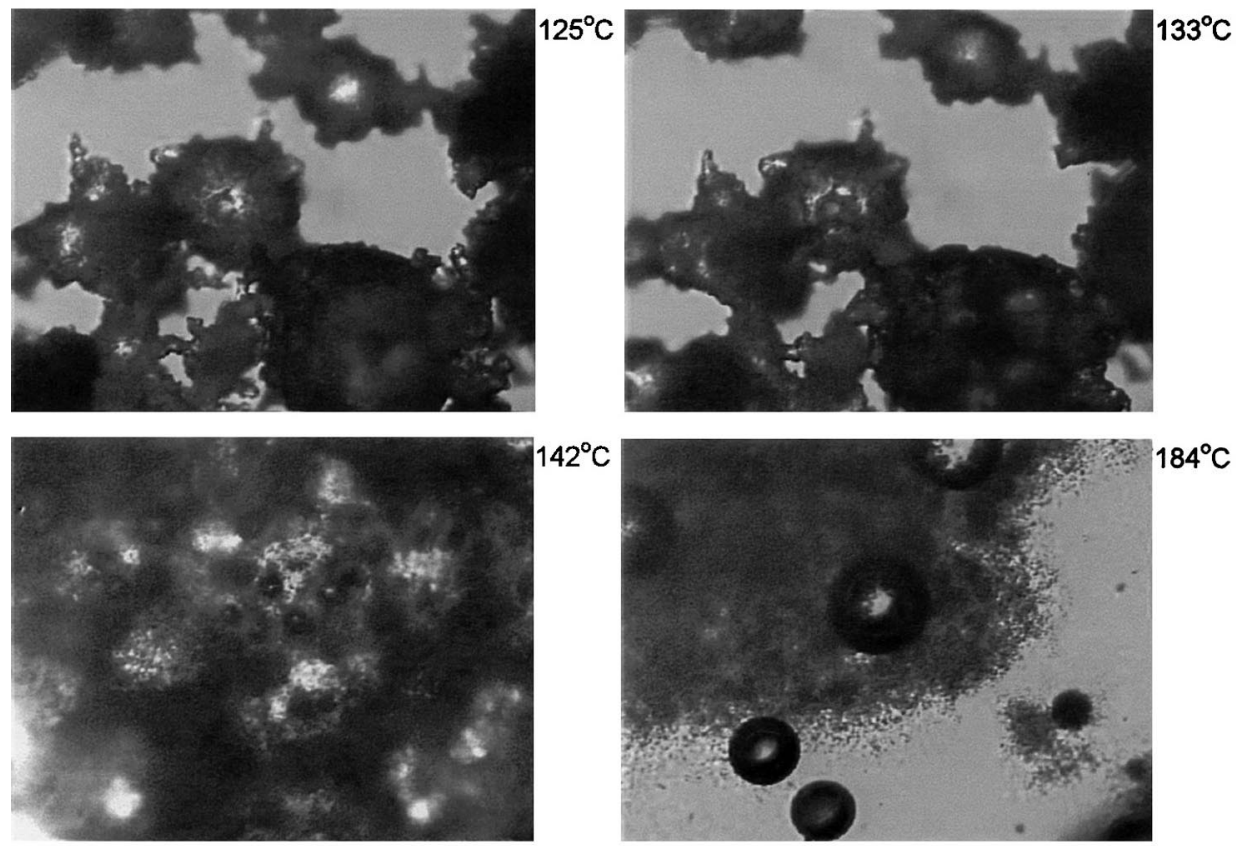

$205^{\circ} \mathrm{C}$

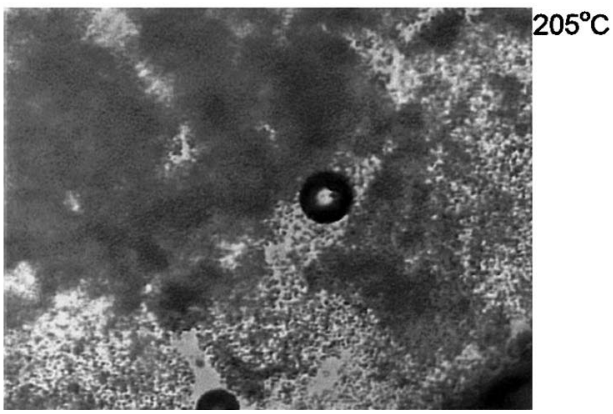

Fig. 3. Typical microscopic views (magnification: $200 \times$ ) of the thermal behavior of the DNAM/PSAN composition $30 / 70$ at $10^{\circ} \mathrm{C} \mathrm{min}^{-1}$.

cally and/or chemically interact, taking into account their known properties.

The thermal stability of pure DNAM can be related with the stability of the crystal lattice, which, in turn, can be understood in the light of the chemical structure of the molecule. The study of DNAM by FT-IR [16] indicates that in the solid state the keto form prevails, for which two tautomeric forms I and II 
are possible:<smiles>O=c1nc(N[N+](=O)[O-])nc2n1[CH]O[N+](=O)N2</smiles>

(I)<smiles>O=c1nc2n3c(n1)N[N+](=O)OC3O[N+](=O)N2</smiles>

(II)
The formation of intra-molecular (see structures I and II) and/or inter-molecular hydrogen bonds are plausible in DNAM. The presence in the crystal of hydrogen bonding is known to be an important stabilizing factor. As mentioned, the decomposition process starts in the condensed phase. Therefore, the close causes of the decomposition of DNAM should be found in some solid (DNAM)/solid (PSAN) interface phenomenon which influences that stabilizing factor in DNAM. Two possible explanations, not necessarily independent, can be postulated: a purely physical effect, involving only polarization effects between the contacting surfaces; or the occurrence of an actual chemical reaction between the two components.

As a consequence of the phase transition, a change in the distribution of electric charges over the surface of PSAN particles may induce a redistribution of electronic density in the molecules of DNAM close to the solid/solid interface. The effect of this redistribution would weaken the order of the crystal system and thus the thermal stability of DNAM. As a consequence, it starts to decompose.

Other possible explanation is the occurrence of a proton transfer reaction. The primary nitramino groups in DNAM are known to be acidic and prone to decomposition through elimination of $\mathrm{N}_{2} \mathrm{O}$. The acidic character of DNAM is confirmed by a $\mathrm{p} K_{\mathrm{a}}$ of ca. 3.2, corresponding to the first dissociation (a three-step curve was found by potentiometric titrating DNAM, in ca. $0.001 \mathrm{~mol} \mathrm{l}^{-1}$ aqueous solution, with $0.1 \mathrm{~mol} \mathrm{l}^{-1} \mathrm{NaOH}$, at room temperature) [16]. On the other hand, when AN is heated two major reactions are involved $[11,17,18]$ : the endothermic dissociation

$\mathrm{NH}_{4} \mathrm{NO}_{3} \rightleftharpoons \mathrm{NH}_{3}+\mathrm{HNO}_{3}$

and the exothermic decomposition

$\mathrm{NH}_{4} \mathrm{NO}_{3} \rightarrow \mathrm{N}_{2} \mathrm{O}+2 \mathrm{H}_{2} \mathrm{O}$

The dissociation is the only reaction known to occur below the melting point [19]. The ammonium cation has the ability of a fast proton transfer, but at ambient temperature the equilibrium represented by Eq. (1) is mainly shifted to the left. However, being an endothermic reaction, the dissociation increases with temperature and thus the concentrations of the gaseous species $\mathrm{NH}_{3}$ and $\mathrm{HNO}_{3}$ over the surface of the solid increases. On the other hand, taking into account the presence of acid sites on the surface of DNAM, an interaction between this and PSAN through a proton transfer mechanism may occur. Such a kind of reactions at surfaces is well-known in acid-base chemisorption processes [20]. This may explain a loss of the structural order of DNAM and thus the observed alteration in the reactivity of this component. A corollary of this interpretation arises from the solid/solid interface phenomenon which seems to play a key role: the particle size distribution of each component in the mixture has also to be taken into account in the initiation of the decomposition.

Once initiated, the reactive process is expected to become increasingly complex. The first signs of melting were typically observed near the smallest particles of PSAN in contact with those of DNAM already visibly under decomposition, while perceptible changes in the largest particles were found later, as more liquid mass was accumulated. The visual inspection confirmed an increase in the reactive activity when a solid/liquid mixture was reached, and as the liquid phase was enough to immerse the DNAM particles. The photograph taken at $142^{\circ} \mathrm{C}$ shown in Fig. 3 gives a typical view of such an activity, over which a considerable amount of gas was evolved together with the formation of small crystals, apparently from the DNAM particles. These observations suggest the existence of thermal coupling between the decomposition of DNAM and the melting of PSAN, starting from the formation of the first spots of melt. The liquid assists the exothermic decomposition of DNAM in reaching a self-sustained stage 
which, in turn, becomes an extra source of heat available to melt the remaining solid particles of PSAN, including the largest ones, by the propagation of this co-operative process throughout the overall sample mass. This essentially thermophysical interaction between both components cannot be separated from the thermochemistry of the reactive process, whose interpretation is limited by the lack of deeper experimental evidences regarding the system under study. Nevertheless, some general explanations, necessarily provisional, can be advanced.

As far as PSAN is concerned, there are some indications that the decomposition of this component is facilitated by the presence of DNAM and/or its decomposition products. As can be observed in both Figs. 1 and 2(a), the endothermic peak ascribable to the decomposition of PSAN is shifted to lower temperatures as DNAM content in the mixtures increases. The TG curves in general also show a slight increase and a subsequent decrease in the mass loss rate within the approximate temperature range $180-200^{\circ} \mathrm{C}$, that is, the range within which the onset of the decomposition of PSAN falls in the conditions of the study. Eq. (2) reflects a net reaction and several breakdown mechanisms have been proposed $[5,6,8-$ 11]. For the present discussion, is enough to consider the generally accepted ionic mechanism that prevails at temperatures below $300^{\circ} \mathrm{C}$, according to which the decomposition of $\mathrm{AN}$ is inhibited by both $\mathrm{H}_{2} \mathrm{O}$ and $\mathrm{NH}_{2}$, while $\mathrm{HNO}_{3}$ has a catalytic effect $[9,11]$. Considering that DNAM may have the capability of shifting the acid-base equilibrium (Eq. (1)) in favor of the formation of $\mathrm{HNO}_{3}$, an increase of the rate of the decomposition of PSAN would occur. Unfortunately, the macroscopic character of the TA curves and the absence of further experimental evidences do not allow us to get a deeper insight on the actual influence of DNAM on the decomposition of PSAN.

In has been reported [15] that hydrolysis of DNAM occurs rapidly at $80^{\circ} \mathrm{C}$, with nitrous oxide liberation and the attainment of quantitative yields in cyanuric acid. Despite the different reaction medium (molten PSAN instead of water), the products of DNAM hydrolysis are possible in the case of the thermal decomposition that is being discussed, particularly for the composition range where considerable amount of liquid mass is formed. Assuming the following conversion,

$$
\mathrm{C}_{3} \mathrm{H}_{3} \mathrm{~N}_{7} \mathrm{O}_{5}(\mathrm{~s}) \rightarrow \mathrm{C}_{3} \mathrm{H}_{3} \mathrm{~N}_{3} \mathrm{O}_{3}(\mathrm{~s})+2 \mathrm{~N}_{2} \mathrm{O}(\mathrm{g})
$$

it is possible to compare the theoretical percentage of $\mathrm{N}_{2} \mathrm{O}$ evolved with the percentage of mass loss corresponding to the first stage of the TG curves in Fig. 2(b). The relative differences between the calculated and the measured values (for these, the inflexion point in the TG curves has been used as criterion for defining the end of the first mass loss stage) are within the $\pm 10 \%$ interval for the successive DNAM/PSAN ratios from $10 / 90$ to $60 / 40$. For the remaining compositions substantially larger differences were found. The possibility of these results being merely fortuitous cannot be neglected. Nevertheless, mention should be made for further results presented below. These, although insufficient to prove the occurrence of Eq. (3), indicate its likelihood or, at least, that the decomposition of DNAM in molten PSAN involves mainly the substituent groups of the $s$-triazine ring, while the ring resists to cleavage during the process. Fig. 4(a) shows the thermal behavior of pure cyanuric acid. This starts to melt with decomposition at about $325^{\circ} \mathrm{C}$. Fig. 4(b) presents the TA curves of a mixture of PSAN and cyanuric acid. The thermal decomposition ascribable to each component in the mixture follows a similar trend to the observed when each component is studied alone. This suggests that the thermal behavior of cyanuric acid is not strongly influenced by the thermal decomposition of PSAN, and thus that any possible interaction between both components when mixed should be small. Fig. 4(c) presents the TA curves for the indicated DNAM/PSAN mixture, whose study was extended up to $500^{\circ} \mathrm{C}$. A broad endothermic peak starting at approximately $360^{\circ} \mathrm{C}$ is observed. This peak appears somehow shifted to higher temperatures when compared with the peak due to the melting/ decomposition of cyanuric acid either pure or mixed with PSAN. However, this does not exclude the possibility of being the last endothermic peak in Fig. 4(c) ascribable to cyanuric acid and/or some homologous compound. It is well-known that the melting behavior, which in the case of cyanuric acid cannot be separated from the concomitant decomposition, can be strongly influenced by factors such as the thermal history of the sample and/or its crystal size. But even discharging this possibility, these results indicate that the endother- 


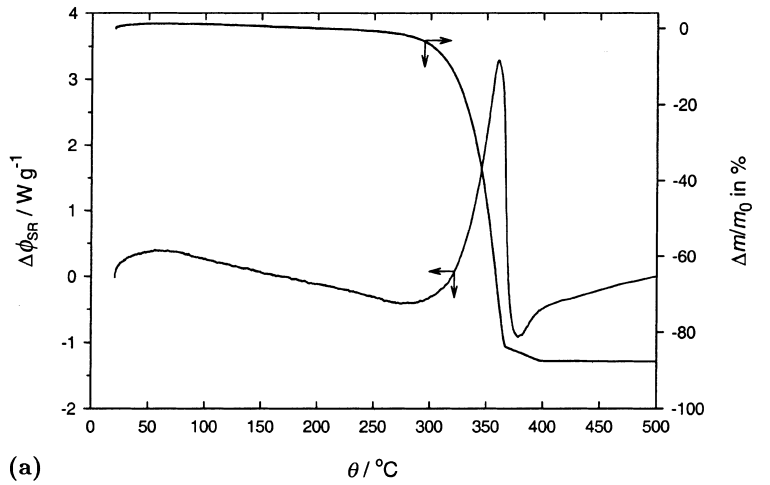

(a)

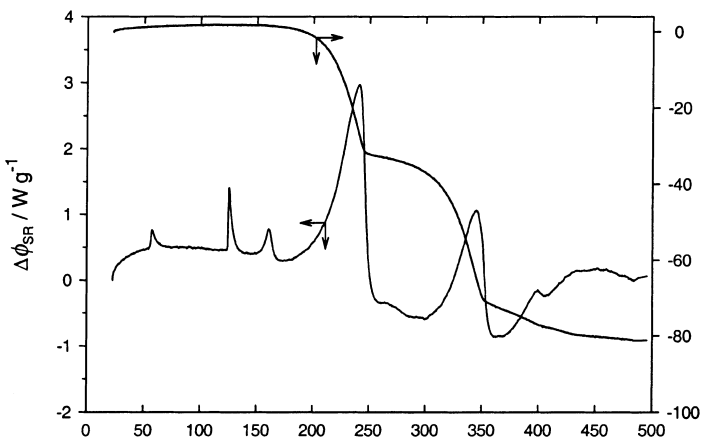

(b)

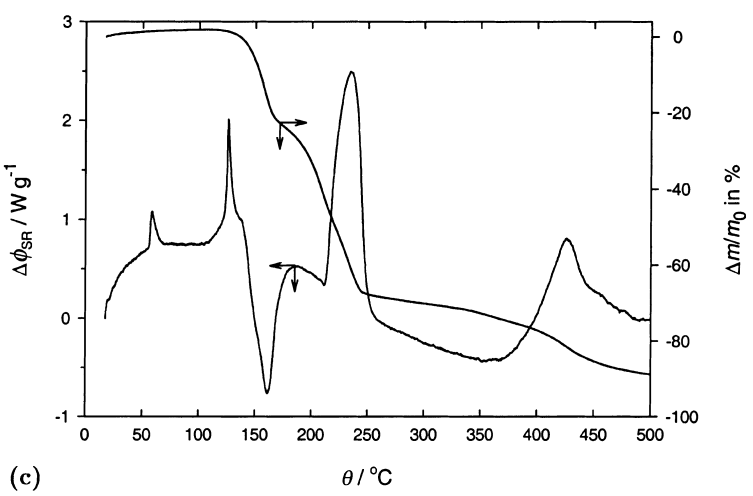

Fig. 4. TA curves of (a) cyanuric acid, (b) mixture of PSAN/ cyanuric acid mixture (mass ratio 50/50), and (c) DNAM/PSAN mixture (mass ratio 50/50) at $10^{\circ} \mathrm{C} \mathrm{min}{ }^{-1}$.

mic peak observed at higher temperatures in the DNAM/PSAN mixture should be attributed to some $s$-triazine by-product, meaning that the decomposition of DNAM is predominantly confined to the $s$-triazine substituent groups (possibly the nitramino groups). It is interesting to note that melamine (2,4,6-triamine-striazine), of which DNAM is the nitrated derivative, also starts to decompose at about $360^{\circ} \mathrm{C}$ under experimental conditions comparable to those used in our study.

\subsection{Kinetic analysis}

DNAM/PSAN ratios 50/50 and 60/40 were selected for measurements at different heating rates for a reliable kinetic analysis. Their choice was based on the propellant chemistry considering the methods for calculating thermochemical parameters of fuel-oxidizer ratio [14]. Fig. 5 presents typical plots of $\alpha$ and $\mathrm{d} \alpha /$ $\mathrm{d} t$ as a function of temperature obtained from the TG curves after normalization (first stage only). The onset of the second stage was used to define the limit of the mass loss for the normalization. The apparent values of the activation energy of the decomposition process have been calculated according to the extended Friedman method [21], which starts from the basic equation

$\frac{\mathrm{d} \alpha}{\mathrm{d} t}=A \exp \left(-\frac{E}{R T}\right) f(\alpha)$

leading to

$\ln \left(\frac{\mathrm{d} \alpha}{\mathrm{d} t}\right)=\ln [A f(\alpha)]-\frac{E}{R T}$

where $\alpha$ is the degree of conversion, $E$ the activation energy, $A$ the pre-exponential factor, $T$ the absolute temperature, $R$ the gas constant, $t$ the time, and $f(\alpha)$ is the kinetic model function based on the physicogeometric assumption of the reaction interface movement. This method allows the estimation of $E$ without any assumption other than the rate of the process being described by the Arrhenius law [22,23].

Fig. 6 presents the plots of $E$ against $\alpha$ for both compositions. For composition 50/50 the value of $E$ is approximately constant with $\alpha$ over almost all the range studied, while for the mixture 60/40 a more pronounced variation is observed near the extremes of $\alpha$. The variation of $E$ near the lower conversion limit may be attributed to the thermal effects arising from the coexistence of the exothermic decomposition of DNAM and the solid/solid endothermic transition of PSAN occurring immediately after the very beginning of the reactive process. On the other hand, the opposite trends of the variation of $E$ with $\alpha$ in the upper limit 

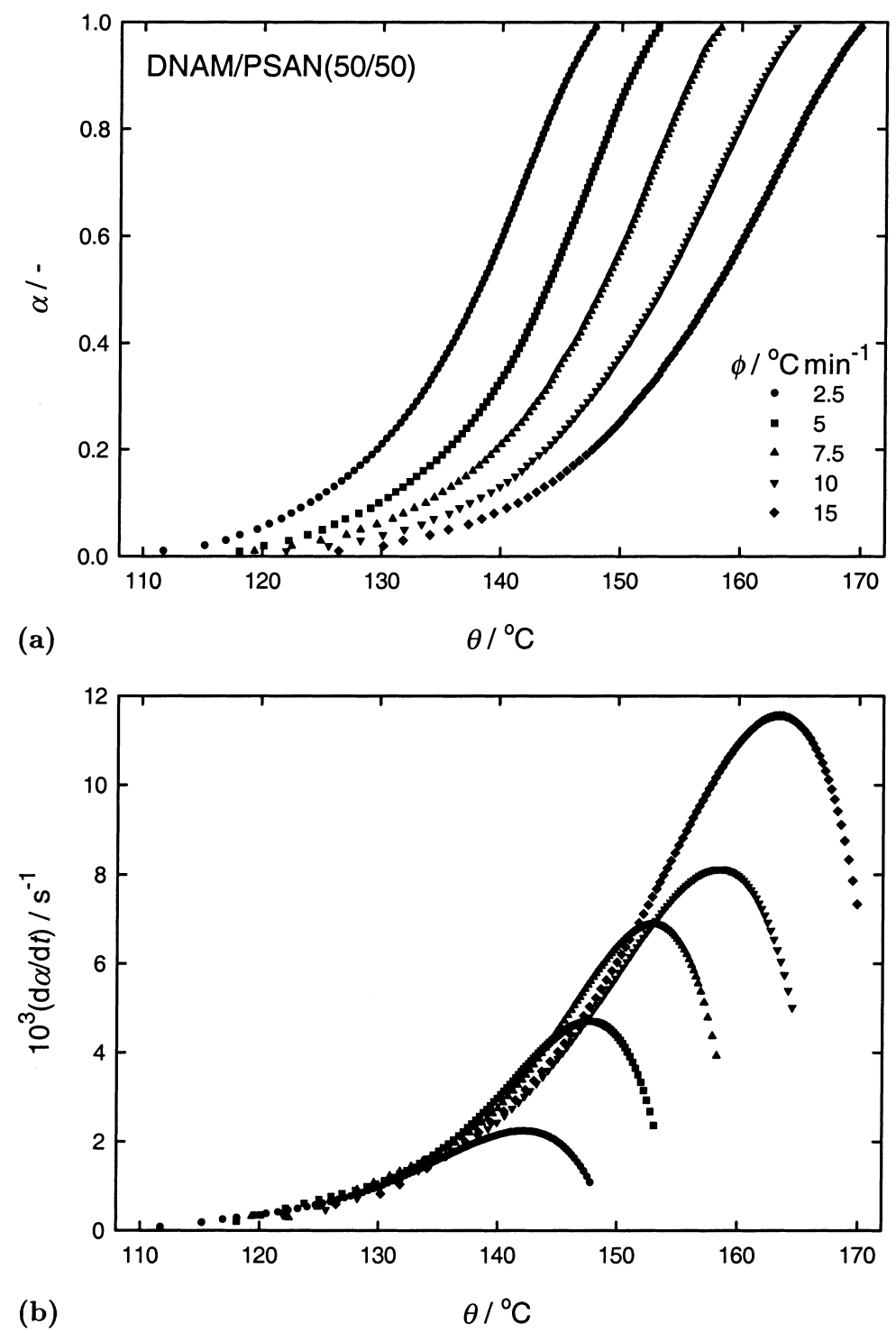

Fig. 5. Typical data derived from TG curves to be used in the kinetic analysis: (a) $\alpha$ and (b) $d \alpha / d t$ as a function of temperature.

of conversion depending on the composition may indicate different pathways in the decomposition mechanisms after the first stage.

The conversion values at the maximum rate of conversion, $\alpha_{\mathrm{p}}$, are presented in Table 1 . Since these values are approximately independent of the heating rate, we admit that the rate constant can be modeled by the Arrhenius law [23]. This, together with the reason- able constancy of $E$ with $\alpha$ allow us to proceed with the kinetic analysis by using a mean value of $E$.

The mean values of $E$ presented in Table 2 have been calculated from Fig. 6, for each composition, for $\alpha$ from 0.20 to 0.90 , satisfying the criterion of a deviation from the mean within the interval of $\pm 10 \%$.

Introducing the concept of generalized time, $\tau$, proposed by Ozawa [24-26], the reaction rate at 


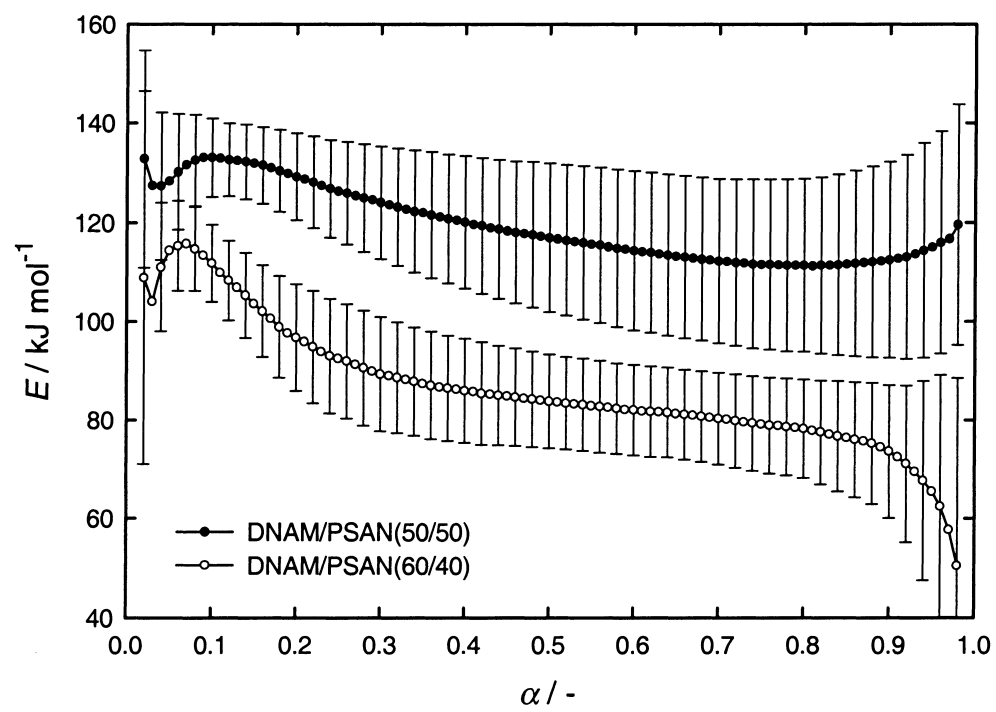

Fig. 6. Activation energy as a function of the degree of conversion calculated from the Friedman method.

infinite temperature [27] is obtained as

$\frac{\mathrm{d} \alpha}{\mathrm{d} \tau}=\frac{\mathrm{d} \alpha}{\mathrm{d} t} \exp \left(\frac{E}{R T}\right)$

or

$\frac{\mathrm{d} \alpha}{\mathrm{d} \tau}=A f(\alpha)$
The value of $A$ can be obtained from the slope of the plot of $\mathrm{d} \alpha / \mathrm{d} \tau$ against $f(\alpha)$. However, to avoid a possible distortion of $A$ due to a wrong choice of $f(\alpha)$, which is unknown a priori, the most adequate kinetic model function describing the process should be identified independently. Therefore, it can be useful to resort to empirical functions, $h(\alpha)$, involving a number of parameters as low as possible. Two commonly used

Table 1

Values of $\alpha_{\mathrm{p}}$ and $\alpha_{\mathrm{M}}$, and of the calculated model parameters

\begin{tabular}{|c|c|c|c|c|c|c|c|c|c|}
\hline \multirow[t]{2}{*}{ DNAM/PSAN } & \multirow[t]{2}{*}{$\phi\left({ }^{\circ} \mathrm{C} \min ^{-1}\right)$} & \multirow[t]{2}{*}{$\alpha_{\mathrm{p}}$} & \multirow[t]{2}{*}{$\alpha_{M}$} & \multicolumn{4}{|l|}{$\mathrm{SB}(M, N)^{\mathrm{a}}$} & \multicolumn{2}{|l|}{$\mathrm{JMA}(M)^{\mathrm{b}}$} \\
\hline & & & & $M$ & $N$ & $r^{\mathrm{c}}$ & $r^{\mathrm{d}}$ & $M$ & $r^{\mathrm{d}}$ \\
\hline \multirow[t]{5}{*}{$50 / 50^{\mathrm{e}}$} & 2.5 & $0.72 \pm 0.01^{\mathrm{f}}$ & $0.35 \pm 0.02$ & $0.29 \pm 0.02$ & $0.53 \pm 0.00$ & 0.9985 & 0.9991 & $1.78 \pm 0.08$ & 0.9987 \\
\hline & 5 & $0.73 \pm 0.02$ & $0.44 \pm 0.02$ & $0.40 \pm 0.02$ & $0.51 \pm 0.02$ & 0.9926 & 0.9960 & $2.39 \pm 0.20$ & 0.9970 \\
\hline & 7.5 & $0.74 \pm 0.01$ & $0.40 \pm 0.02$ & $0.35 \pm 0.03$ & $0.53 \pm 0.01$ & 0.9975 & 0.9976 & $2.05 \pm 0.14$ & 0.9993 \\
\hline & 10 & $0.73 \pm 0.00$ & $0.32 \pm 0.02$ & $0.25 \pm 0.02$ & $0.54 \pm 0.01$ & 0.9985 & 0.9984 & $1.62 \pm 0.06$ & 0.9995 \\
\hline & 15 & $0.74 \pm 0.01$ & $0.24 \pm 0.01$ & $0.16 \pm 0.01$ & $0.53 \pm 0.00$ & 0.9994 & 0.9990 & $1.37 \pm 0.02$ & 0.9997 \\
\hline \multirow[t]{5}{*}{$60 / 40^{\mathrm{g}}$} & 2.5 & $0.69 \pm 0.00$ & $0.36 \pm 0.01$ & $0.29 \pm 0.01$ & $0.52 \pm 0.01$ & 0.9985 & 0.9991 & $1.81 \pm 0.07$ & 0.9992 \\
\hline & 5 & $0.73 \pm 0.02$ & $0.48 \pm 0.03$ & $0.45 \pm 0.01$ & $0.49 \pm 0.05$ & 0.9882 & 0.9931 & $2.93 \pm 0.46$ & 0.9927 \\
\hline & 7.5 & $0.72 \pm 0.01$ & $0.46 \pm 0.01$ & $0.43 \pm 0.03$ & $0.50 \pm 0.01$ & 0.9899 & 0.9964 & $2.61 \pm 0.18$ & 0.9963 \\
\hline & 10 & $0.71 \pm 0.02$ & $0.44 \pm 0.04$ & $0.40 \pm 0.03$ & $0.51 \pm 0.05$ & 0.9992 & 0.9989 & $2.43 \pm 0.44$ & 0.9996 \\
\hline & 15 & $0.72 \pm 0.01$ & $0.43 \pm 0.04$ & $0.38 \pm 0.04$ & $0.51 \pm 0.04$ & 0.9864 & 0.9963 & $2.32 \pm 0.40$ & 0.9967 \\
\hline
\end{tabular}

${ }^{\mathrm{a}} h(\alpha)=\alpha^{M}(1-\alpha)^{N}$.

${ }^{\mathrm{b}} h(\alpha)=M(1-\alpha)[-\ln (1-\alpha)]^{1-1 / M}$.

${ }^{c}$ Worst regression coefficient among those obtained by applying Eq. (8) to data from each run.

${ }^{\mathrm{d}}$ Worst regression coefficient among those obtained by applying Eq. (7) to data from each run.

e Three runs per heating rate.

${ }^{\mathrm{f}}$ Standard deviation.

${ }^{\mathrm{g}}$ Two runs per heating rate. 
Table 2

Arrhenius parameters of the thermal decomposition of pure DNAM and DNAM/PSAN mixtures

\begin{tabular}{lllll}
\hline Reference & Sample & $E\left(\mathrm{~kJ} \mathrm{~mol}^{-1}\right)$ & $\log A\left(\mathrm{~s}^{-1}\right)$ & $\Delta \theta^{\mathrm{a}}\left({ }^{\circ} \mathrm{C}\right)$ \\
\hline$[1]$ & DNAM & 269 & 26.4 & $220-235$ \\
This work & DNAM/PSAN & & & \\
& $50 / 50$ & $117 \pm 14$ & $12.5 \pm 0.1$ & $110-170$ \\
& $60 / 40$ & $84 \pm 10$ & $8.2 \pm 0.1$ & $110-175$ \\
\hline
\end{tabular}

${ }^{\text {a }}$ Approximate temperature range of the kinetic analysis.

empirical functions are the Sestak-Berggren (SB) [28] and Johnson-Mehl-Avrami (JMA) [29,30].

It has been reported [31,32] that the shape of the plot of $\mathrm{d} \alpha / \mathrm{d} \tau$ against $\alpha$ as well as the value of $\alpha_{M}$ that corresponds to the conversion at the maximum, if exists, are of importance as a diagnostic tool to identify the most probable form of $h(\alpha)$. A concave shape in both compositions under analysis was identified, meaning that $\alpha_{M} \neq 0$ and making the use of SB or JMA empirical models possible [31,32]. In spite of the existence of a common pattern for both compositions, it is noticeable from Table 1 that the values of $\alpha_{M}$ are not constant with the heating rate, being the variation significantly more pronounced for the composition $50 / 50$.
The kinetic exponents $M$ and $N$ in the SB model were evaluated according to the relationship [32]

$\ln \left(\frac{\mathrm{d} \alpha}{\mathrm{d} \tau}\right)=\ln A+N \ln \left[\alpha^{P}(1-\alpha)\right]$

Fig. 7 shows a typical plot of fitting, being the results for all cases summarized in Table 1. For the JMA model, the exponent $M$ was obtained according to [32]

$M=\frac{1}{1+\ln \left(1-\alpha_{\mathrm{M}}\right)}$

The results are also resumed in Table 1 . The reliability of the regression and the quality of the fitting were checked by a residual analysis and the determination of the correlation coefficients. The variation of the

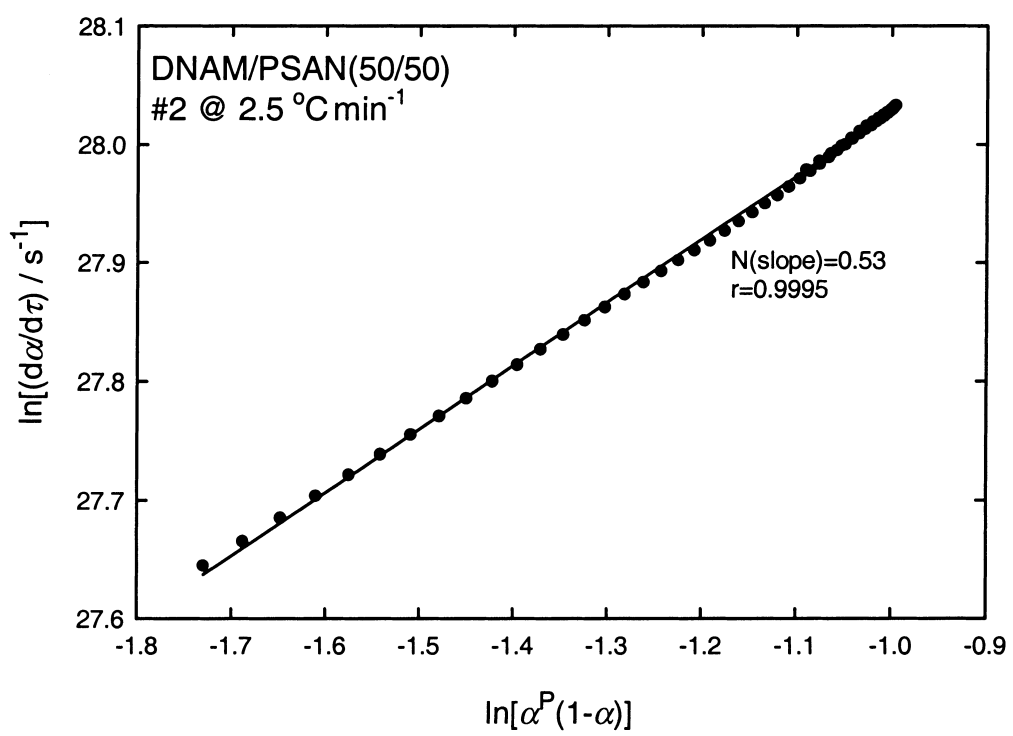

Fig. 7. Typical plot to determine the parameter $N$ of the $\mathrm{SB}(M, N)$ model by linear regression according to Eq. (7). 


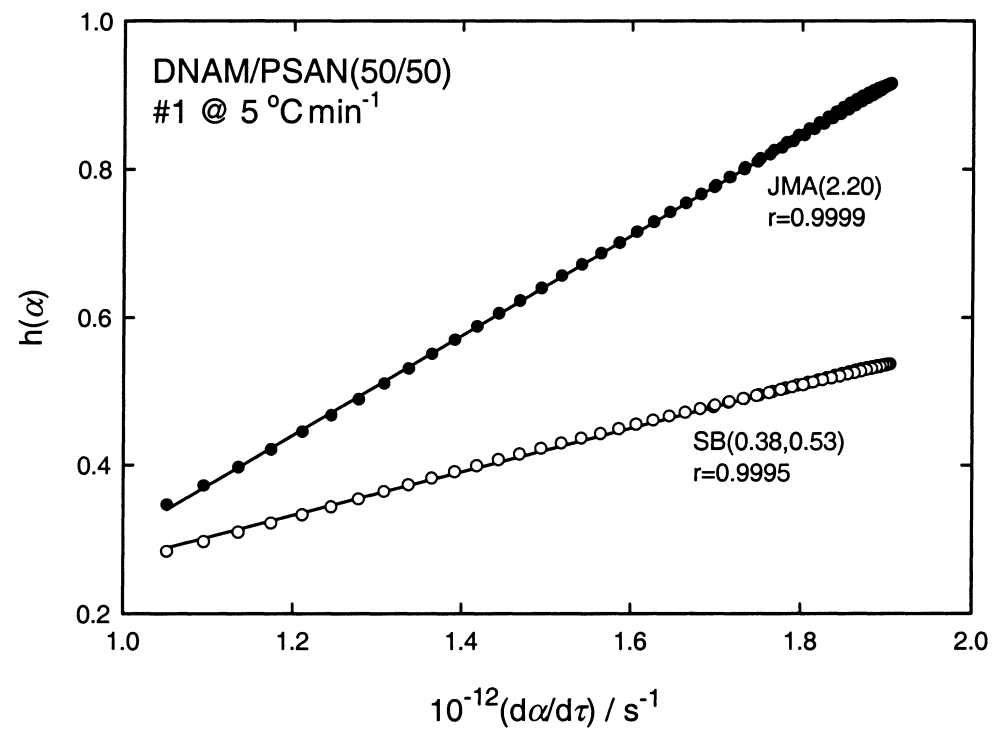

Fig. 8. Typical plot to check the possible adequacy of $\operatorname{SB}(M, N)$ and $\operatorname{JMA}(M)$ models, within the restricted range of kinetic analysis, by applying Eq. (7).

kinetic exponents of each model is obviously related with the variation of $\alpha_{M}$ with the heating rate.

In order to check the adequacy of the models to describe the process we plotted $\mathrm{d} \alpha / \mathrm{d} \tau$ against $h(\alpha)$ in the restricted range of $\alpha$ of the kinetic analysis $(0.20 \leq \alpha \leq 0.90)$ according to Eq. (7). This has been done considering the results from each heating rate separately, and a typical plot is presented in Fig. 8. This example, together with the correlation coefficients obtained in the remaining cases (see Table 1), indicate that both models may be valid to describe the decomposition process under study. However, the use of plots as illustrated in Fig. 8 may lead to erroneous conclusions, since no check the invariance of $A$ with $\alpha$ within the range of kinetic analysis is made. This is confirmed in Fig. 9 where it is possible to observe that only for the SB model a constant value of $A$ was obtained. Despite the good linearity exhibited by the JMA model as shown in Fig. 8, the variation of $A$ with $\alpha$ reveals its inadequacy to describe the process.

For a final check of the consistency of the kinetic analysis, the constant values of $A$ have been used to plot $\mathrm{d} \alpha /(A \mathrm{~d} \tau)$ against $\alpha$ and then compared with the correspondent mathematically drawn function $\mathrm{SB}(M, N)$. Fig. 10 shows a typical example of the excellent agreement between the experimental points and the calculated curves obtained in the range of the kinetic analysis. A typical plot of $\mathrm{d} \alpha / \mathrm{d} t$ against $\alpha$ values obtained from the experimental data, confronted with the values calculated from Eq. (4) using the estimated Arrhenius parameters and replacing $f(\alpha)$ by the empirical $\mathrm{SB}(M, N)$ function is also presented in Fig. 10.

The SB describes the real process closely. The sensitivity of the model to the features of the process, such as the effect of the heating rate, has been evidenced by the variation of the kinetic exponents. This clearly shows the flexibility of the SB empirical function in describing several shapes of the $d \alpha / d \tau$ against $\alpha$ curves satisfying the condition $\alpha_{M} \neq 0$. However, some limitations of the model due to its purely empirical character are to be accounted. Unfortunately, it is extremely difficult or even impossible to give a physical meaning to the kinetic exponents $M$ and $N$ of the SB model. Thus, no theoretical model based on physico-geometric assumptions of the reaction interface movements were found to describe our experimental data, probably as a consequence of the complexity of the process under study. Nevertheless, this limitations are not critical considering the comparative nature of the kinetic study performed here.

The apparent values of $A$ for each DNAM/PSAN composition studied are resumed in Table 2. They have been obtained from the mean values found at 


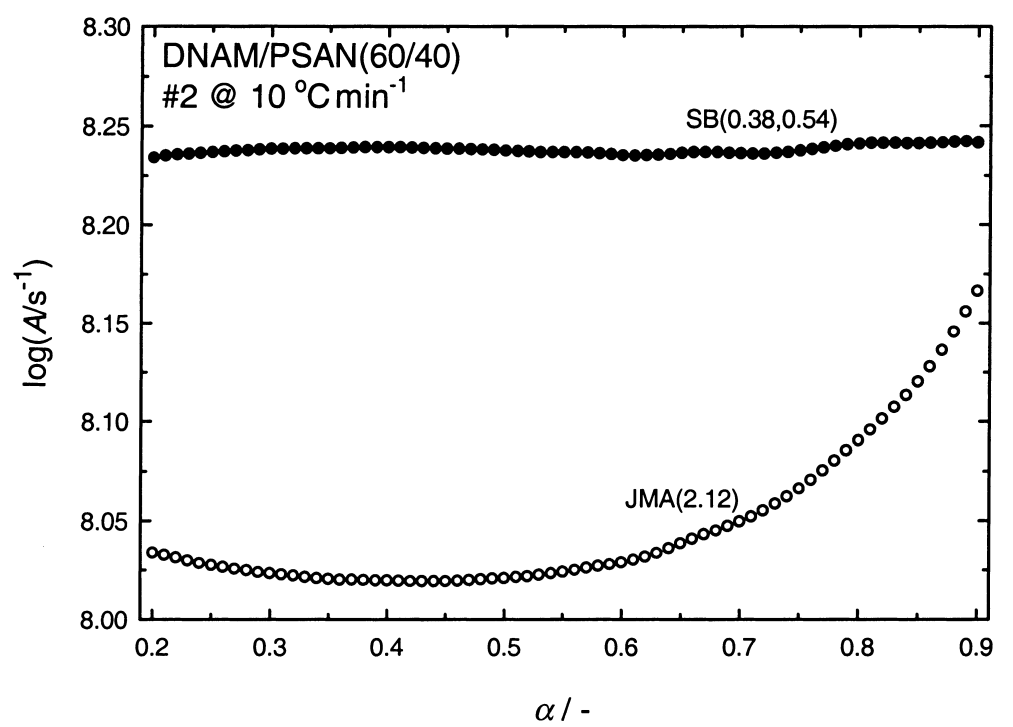

Fig. 9. Typical plot to determine $A$ and to check its constancy with $\alpha$, within the restricted range of kinetic analysis, by applying logarithms to Eq. (7) and by using the models previously determined.

each heating rate. In a strict sense, this procedure might be susceptible of some criticism, since the kinetic obedience to an unique function $h(\alpha)$ over the heating rates applied is not strictly fulfilled. However, it was found that the values of $A$ were not strongly influenced by the variation of the kinetic exponents $M$ and $N$ with the heating rate. Furthermore, the good linearity obtained by applying Eq. (5) at different heating rates in the determination of $E$ gives the necessary consistency to the kinetic analysis.

The results in Table 2 support in a quantitative basis some of the conclusions previously drawn from the

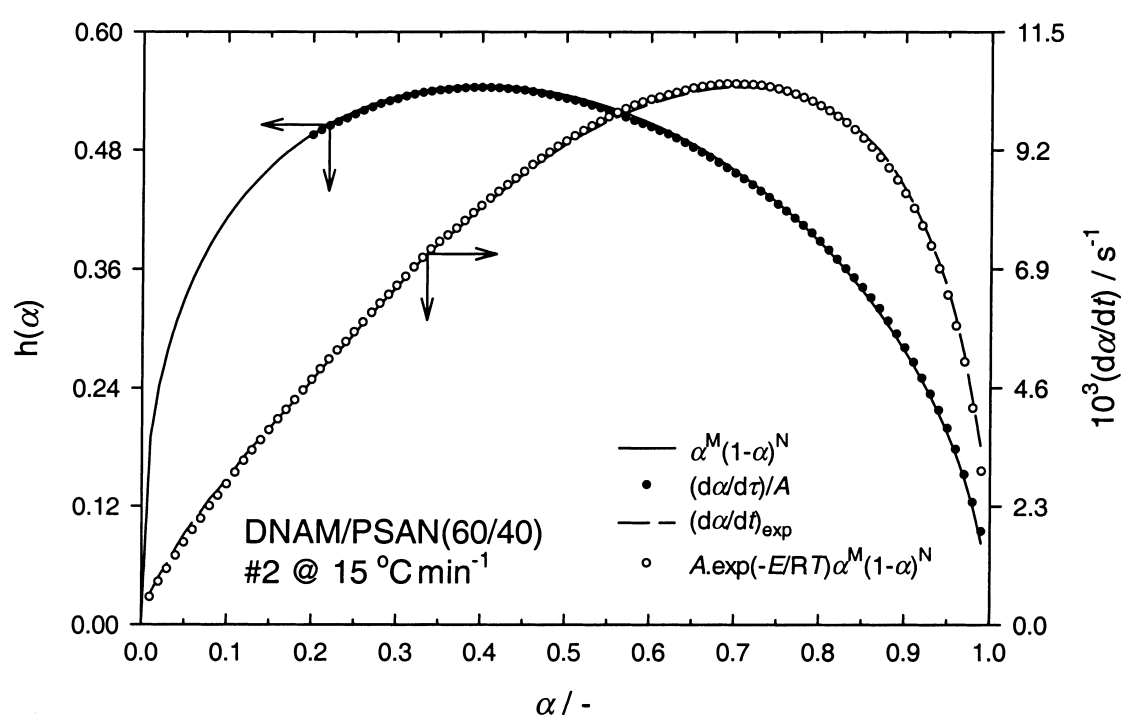

Fig. 10. Typical plot to check the adequacy of the Arrhenius parameters and of the $\operatorname{SB}(M, N)$ in describing the process within the restricted range of kinetic analysis. 
interpretation of the TA curves as well as from the thermomicroscopy observations. Remembering that DNAM is the component that starts to decompose in the mixtures, the corresponding Arrhenius parameters presented in Table 2 should be ascribed to the decomposition of DNAM in the presence of PSAN. Whatever the composition, clear departures from the values of $E$ and $A$ corresponding to the case of pure DNAM are observed. As a consequence of the shift of the decomposition temperature range as well as the physical and chemical environmental of its occurrence, changes in the decomposition mechanism of DNAM are likely especially after comparison with what happens with pure DNAM.

The substantial differences in the Arrhenius parameters of the decomposition of the two mixtures confirm important changes in the thermal behavior of the mixtures in that composition range. This means that near the DNAM/PSAN stoichiometric mixture the thermochemistry of the decomposition process is rather sensitive to the relative amounts of the two components, which is a factor deserving attention from a practical point of view in the development of DNAM/PSAN based propellants.

\section{Conclusion}

The study carried out on the thermal decomposition of solid mixtures of DNAM and PSAN allowed us to conclude that the decomposition process starts in the solid state. DNAM is the component that starts to decompose at temperatures close to the temperature of the solid/solid phase transition II $\rightarrow$ I of PSAN. These observations have been discussed in the light of some intrinsic properties of both components. A solid/solid interface phenomenon seems to be responsible by the loss of the structural order of DNAM crystal system and consequently by the change in the thermal reactivity of this component. Such an interaction can be interpreted either by electric charge effects over the contacting surfaces or by a proton transfer mechanism between the components of the mixture. The TA curves indicate that once initiated the reactive process, the presence of DNAM (and probably of its decomposition products) facilitates the decomposition of PSAN, possibly through the influence on the dissociation of PSAN which is kinetically coupled with the decomposition. On the other hand, the molten PSAN provides a medium for the decomposition of DNAM with a possible distinct mechanism of the thermolysis of pure DNAM. A hypothetical route for the decomposition of DNAM when mixed with an amount enough of PSAN (DNAM/PSAN mass ratios from $10 / 90$ to $60 / 40$ ) involving the formation of cyanuric acid and/or some related compound may be advanced in the light of some of the experimental evidences available. However, it is felt that further experimental evidences are needed.

The kinetic analysis performed show that, for the compositions under question, the first step of the decomposition process is acceptably described by the SB kinetic model function. Despite the limitations due to the purely empirical character of the model, the global kinetic treatment permitted to quantitatively substantiate, at least on a comparative basis, some explanations advanced for the process. Furthermore, for DNAM/PSAN ratios close to the stoichiometric mixture the thermochemistry of the decomposition process is rather sensitive to the composition. Such a sensitivity needs to be underlined in terms of both the safety and combustion behavior. From a practical point of view, these aspects are of prime importance within the framework of formulations comprising the DNAM/PSAN pair as the main oxidizer charge.

\section{Acknowledgements}

The authors wish to thank Prof. J. Redinha and coworkers of the Chemistry Department of FCT/UC for their kind assistance with the thermomicroscopy technique.

\section{References}

[1] P. Simões, A. Portugal, J. Campos, Thermochim. Acta 298 (1997) 95.

[2] P. Simões, P. Carvalheira, L. Durães, A. Portugal, J. Campos, Proceedings of the 22nd International Pyrotechnics Seminar, Fort Collins, CO, USA, 15-19 July 1996, pp. 389-396.

[3] P. Simões, P. Carvalheira, A. Portugal, J. Campos, L. Durães, J. Góis, Proceedings of the 27th International Annual Conference of ICT, Karlsrhue, Federal Republic of Germany, 25-28 June 1996, pp. 136/1-136/13. 
[4] N. Koga, H. Tanaka, Thermochim. Acta 240 (1994) 141.

[5] V.A. Koroban, Y.N. Burtsev, F.R. Alimov, A.D. Haustov, V.A. Dubovik, V.A. Teselkin, Propellants, Explosives and Pyrotechnics 19 (1994) 307.

[6] T.B. Brill, P.J. Brush, D.G. Patil, Combustion and Flame 92 (1993) 178.

[7] N. Koga, H. Tanaka, Thermochim. Acta 209 (1992) 127.

[8] D.G. Patil, S.R. Jain, T.B. Brill, Propellants, Explosives and Pyrotechnics 17 (1992) 99.

[9] K.R. Brower, J.C. Oxley, M. Tewari, J. Phys. Chem. 93 (1989) 4029.

[10] T.P. Russell, T.B. Brill, Combustion and Flame 76 (1989) 393.

[11] W.A. Rosser, S.H. Inami, H. Wise, J. Phys. Chem. 67 (1963) 1753.

[12] P.N. Simões, L.M. Pedroso, A.A. Portugal, J.L. Campos, Thermochim. Acta 319 (1998) 55.

[13] P. Carvalheira, G.M.H.J.L. Gadiot, W.P.C. Klerk, Thermochim. Acta 269/270 (1995) 273.

[14] S.R. Jain, K.C. Adiga, V.R.P. Verneker, Combustion and Flame 40 (1981) 71.

[15] E.R. Atkinson, J. Am. Chem. Soc. 73 (1951) 4443.
[16] P. Simões, L. Pedroso, P. Carvalheira, J. Campos, A. Portugal, Propellants, Explosives, Pyrotechnics, 2000, submitted for publication.

[17] B.J. Wood, H. Wise, J. Chem. Phys. 23 (1955) 693.

[18] G. Feick, R.M. Hainer, J. Am. Chem. Soc. 76 (1954) 5860.

[19] T. Urbanski, Chemistry and Technology of Explosives, 2nd Edition, Vol. 2, Pergamon Press, Oxford, 1990.

[20] A.W. Adamson, Physical Chemistry of Surfaces, 5th Edition, Wiley, New York, 1990.

[21] H.L. Friedman, J. Polym. Sci. C6 (1964) 183.

[22] T. Ozawa, J. Thermal Anal. 31 (1986) 547.

[23] T. Ozawa, Thermochim. Acta 203 (1992) 159.

[24] T. Ozawa, Thermochim. Acta 100 (1986) 109.

[25] T. Ozawa, Bull. Chem. Soc. Jpn. 38 (11) (1965) 1881.

[26] T. Ozawa, J. Thermal Anal. 2 (1970) 301.

[27] N. Koga, Thermochim. Acta 258 (1995) 145.

[28] J. Sesták, B. Berggren, Thermochim. Acta 3 (1971) 1.

[29] W.A. Johnson, R.F. Mehl, Trans. Am. Instit. Min. (Metallogr.) Eng. 135 (1939) 416.

[30] M. Avrami, J. Phys. Chem. 9 (1941) 177.

[31] N. Koga, J. Málek, Thermochim. Acta 282/283 (1996) 69.

[32] J. Málek, Thermochim. Acta 200 (1992) 257. 